Schulte, P., Zeil, J. \& Stürzl, W. Biol Cybern (2019). https://doi.org/10.1007/s00422-019-00800-1

\title{
An Insect-Inspired Model for Acquiring Views for Homing
}

\author{
Patrick Schulte • Jochen Zeil • Wolfgang Stürzl
}

Received: date / Accepted: date

\begin{abstract}
Wasps and bees perform learning flights when leaving their nest or food locations for the first time during which they acquire visual information that enables them to return successfully. Here we present and test a set of simple control rules underlying the execution of learning flights that closely mimic those performed by ground-nesting wasps. In the simplest model we assume that the angle between flight direction and the nest direction as seen from the position of the insect is constant and only flips sign, when pivoting direction around the nest is changed, resulting in a concatenation of piece-wise defined logarithmic spirals. We then added characteristic properties of real learning flights, such as head saccades and the condition that the nest entrance within the visual field is kept nearly constant to describe the development of a learning flight in a headcentered frame of reference assuming that the retinal position of the nest is known. We finally implemented a closed-loop simulation of learning flights based on a small set of visual control rules. The visual input for this model are rendered views generated from 3D reconstructions of natural wasp nesting sites and the retinal nest position is controlled by means of simple templatebased tracking. We show that naturalistic paths can be generated without knowledge of the absolute distance to the nest or of the flight speed. We demonstrate in addition that nest-tagged views recorded during such simulated learning flights are sufficient for a homing
\end{abstract}

P. Schulte, W. Stürzl

Institute of Robotics and Mechatronics, German Aerospace

Center (DLR), Wessling, Germany

E-mail: patrick.schulte@dlr.de, wolfgang.stuerzl@dlr.de

J. Zeil

Research School of Biology, The Australian National Univer-

sity, Canberra, Australia

E-mail: jochen.zeil@anu.edu.au agent to pinpoint the goal, by identifying nest direction when encountering familiar views. We discuss how the information acquired during learning flights close to the nest can be integrated with long-range homing models.

Keywords Visual navigation · Flying insects . Learning flights · Visual servoing · Homing

\section{Introduction}

Bees and wasps perform learning flights at the nest and at food locations during which they acquire visual information that allows them to pinpoint such places of significance on subsequent returns (reviewed in Zeil et al. 1996, 2009; Zeil 2012; Collett and Zeil 2018). The flights have a distinct organization with the insects turning back to face the goal and then (as shown in the example of Fig. 1 a,b) pivoting around it along arcs at increasing distance from the goal (Lehrer 1993; Collett and Lehrer 1993; Zeil 1993a; Collett 1995; Hempel de Ibarra et al. 2009; Collett et al. 2013; Philippides et al. 2013; Riabinina et al. 2014; Stürzl et al. 2016; Robert et al. 2018; Lobecke et al. 2018) and increasing height above ground (Zeil 1993a; Zeil et al. 1996). As they fly along arcs, the insects turn head and body in such a way that the goal location is seen (at least in Cerceris wasps) at relatively constant lateral positions in the left visual field during counter-clockwise arcs and in the right visual field during clockwise arcs (e.g. Zeil 1993a; Stürzl et al. 2016). Counter-turning is not continuous but saccadic with head saccades being followed by body saccades. Head orientation is kept relatively constant between saccades (Zeil et al. 2007, 2009; Boeddeker et al. 2010; Riabinina et al. 2014; Boeddeker et al. 2015; Stürzl et al. 2016) and the retinal position of the nest is visually controlled (Zeil 1993a; Samet et al. 2014). 

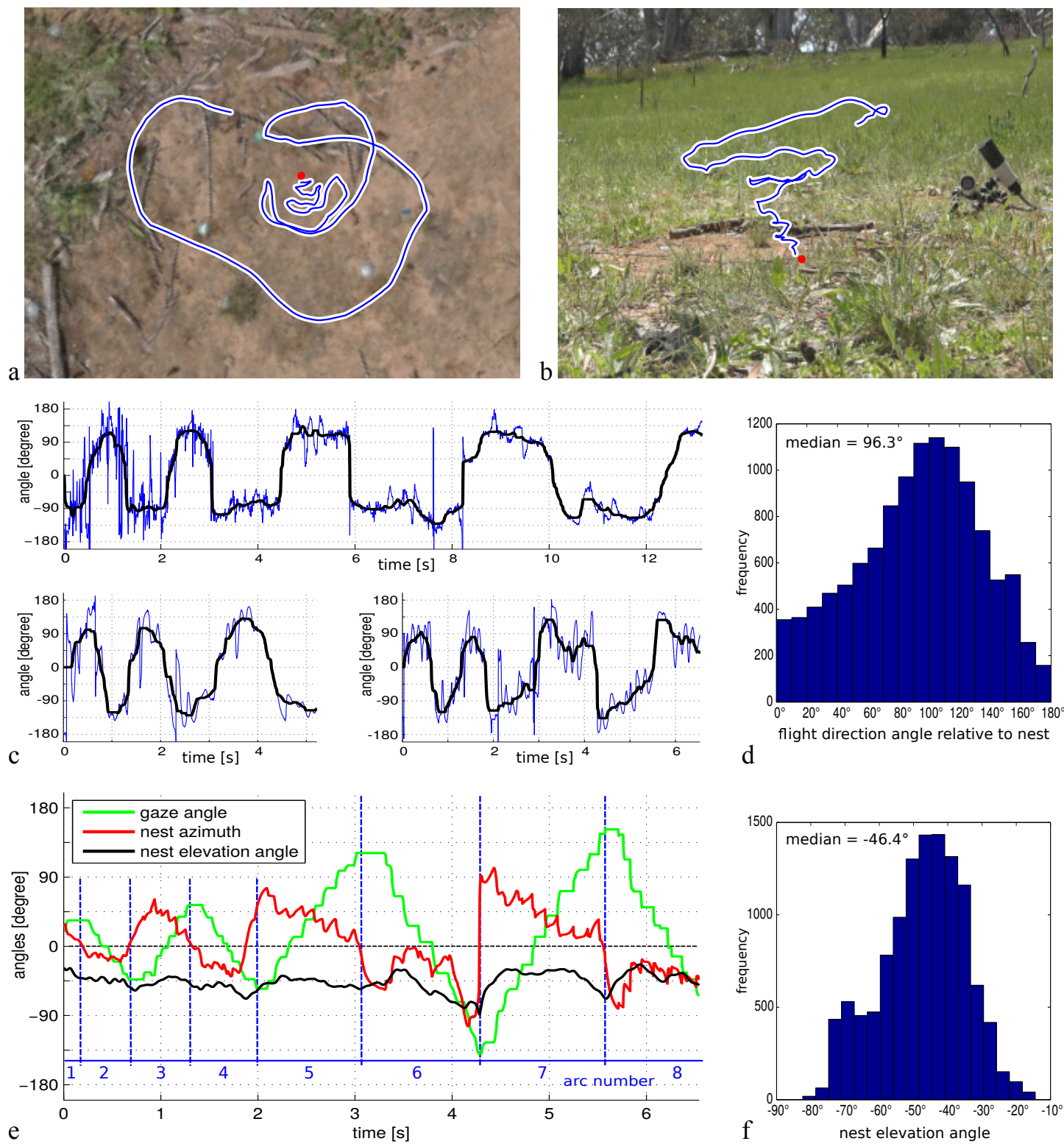

Fig. 1 Examples and characteristic properties of learning flights performed by Cerceris wasps. a,b) Top-down and side view of the first 8 arcs of a learning flight. 2D projections of the insect's path are shown overlayed on camera images. The position of the nest is indicated by a red dot. c) Flight direction relative to the nest $\delta$ of three recorded learning flights. Thick black curves show median-filtered values. Note that the "raw" flight direction angle values were computed from 3D positions estimated every $4 \mathrm{~ms}$ from high-speed stereo videos. The resulting values are noisy, in particular close to the nest, where movement between frames is small. d) Distribution of the absolute value of the flight direction angle $|\delta|$ based on data from 7 learning flights. e) Time-course of the gaze angle (green) as well as the nest azimuth (red) and elevation angle (black) for the first 8 arcs of a learning flight. The beginning and end of each arc is indicated by blue dashed vertical lines. For most flights, the range of the gaze angle increases with number of arcs, while the nest elevation angle stays approximately constant. The nest azimuth is positive for counter-clockwise arcs (nest in left visual field) and negative for clockwise arcs (nest in right visual field). f) Distribution of the nest elevation angle with a peak near $-45^{\circ}$ (data from 7 learning flights).

The execution of learning flights is crucial for successful and efficient homing (e.g. Wagner 1907; Opfinger 1931; Tinbergen 1932; Tinbergen and Kruyt 1938; Zeil 1993b) and their choreography must reflect fundamental constraints of visual navigation and in particular of the acquisition process underlying the navi- gational competence of central place foraging insects. However, it remains unclear how this learning process is controlled and what is learnt when during its execution. Current suggestions regarding the latter question range from discrete snapshot acquisition at the end of arcs (e.g. Collett 1995; Zeil et al. 1996) or be- 
tween saccadic changes in gaze direction (Stürzl et al. 2016) to the (additional) acquisition of motion parallax cues (Zeil 1993b; Brünnert et al. 1994; Lehrer and Collett 1994; Dittmar et al. 2010; Dittmar 2011; Riabinina et al. 2014; Boeddeker et al. 2015; Lobecke et al. 2018). In natural environments, static snapshots, associated with information on goal direction, are sufficient for successful homing (e.g. Graham et al. 2010; Narendra et al. 2013; Stürzl et al. 2016) but insects clearly also learn non-pictorial cues to the presence and distance of landmarks (Zeil 1993b; Brünnert et al. 1994; Lehrer and Collett 1994; Dittmar et al. 2010; Dittmar 2011) presumably by monitoring the motion parallax generated by both their learning and return flight manoeuvres (Zeil 1993b; Riabinina et al. 2014; Boeddeker et al. 2015; Lobecke et al. 2018).

One way of trying to understand the design principles of learning flights and walks is to attempt to quantify the navigational information they may convey in principle, which has been attempted assuming the acquisition of a series of panoramic views (e.g. Baddeley et al. 2009; Graham et al. 2010; Narendra et al. 2013; Dewar et al. 2014; Stürzl et al. 2015, 2016). However, ideally, the full "information content" of these learning routines can only be assessed (and manipulated) by simulating and modifying their full spatial and temporal dynamics. The ultimate aim would be to implement learning flights on flying platforms which would allow us to simulate and test in natural environments the acquisition and use of visual information for homing.

With this aim in mind, we document here the development of synthetic learning flights based on a set of simple and insect-inspired control rules. Our learning flight model can in future be both modified to understand the within and across species variability of these learning routines and be implemented in simulations and flying platforms with the aim of investigating the functional significance of their choreography for homing performance.

\section{Models of Learning Flights}

In this section we present models for generating learning flight paths with increasing complexity and descriptive power. Important parameters of these models are (see Fig. 2 and Fig. 4)

- $\phi, r, z$ : cylindrical coordinates describing the position of the wasp - more exactly the position of the wasp's head - in the nest-centered coordinate system $(\phi$ : polar angle or azimuth, $r$ : radial distance, $z$ : height);

$-\delta$ : angle of flight direction relative to nest direction;

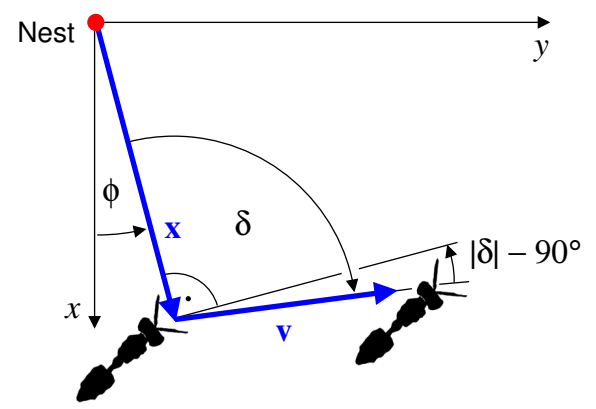

Fig. 2 Illustration of the parameters describing an arc of an idealized learning flight. The flight direction angle $\delta$, the angle between the current flight direction and the wasp-nest-vector $-\mathbf{x}$ determines the path. Note that $\delta<0$ as shown. The nest is at the origin of the coordinate system.

- $\gamma$ : head orientation (yaw angle) in nest-centered coordinate system - the only angle to describe head orientation as we assume that head is stabilized in pitch and roll;

$-\alpha_{\mathrm{H}}$ : retinal azimuth position of the nest in the headcentered coordinate system;

$-\varepsilon_{\mathrm{H}}$ : retinal elevation of the nest in head-centered coordinates;

$-\delta_{\mathrm{H}}$ : angle of flight direction in the head-centered coordinate system.

2.1 Path generation based on constant flight direction angle relative to nest direction

From recorded learning flights one can observe that the absolute value of the flight direction angle with respect to the nest, i.e. the angle between flight direction and the $2 \mathrm{D}$ vector to the nest in the $x-y$-plane is approximately constant while its sign changes quite rapidly with each new arc, see Fig. 1 c,d (Zeil 1993a).

If we simplify these findings by assuming a constant flight direction angle that just flips sign at the beginning of an arc, the path can be calculated as illustrated in Fig. 2. The velocity vector is given by

$\dot{\mathbf{x}}=\mathbf{v}=\lambda \mathbf{R}(\delta)(-\mathbf{x})$,

where $\lambda$ is a scaling factor and $\mathbf{R}(\delta)$ a rotation matrix, describing a rotation by angle $\delta$ around the $z$-axis. As shown in the appendix the solution of the differential equation (1) in polar coordinates with $r=\sqrt{x^{2}+y^{2}}$, $\phi=\arctan 2(y, x)$ is

$$
r=r_{0} \exp \left[\cot \delta\left(\phi-\phi_{0}\right)\right]
$$$$
=r_{0} \exp \left[\tan \left(|\delta|-90^{\circ}\right)\left|\phi-\phi_{0}\right|\right],
$$

describing a "logarithmic spiral". Distance $r$ increases with distance from start angle, $\left|\phi-\phi_{0}\right|$, for $|\delta|>90^{\circ}$. In order to have a sequence of alternating clockwise and counter-clockwise arcs as in real flights, we make angle 
$\phi$ a function of time and change the sign of the flight direction angle $\delta$ with each new arc but keep its absolute value, i.e. we use $\delta=-\delta_{0}<0, \phi(t)-\phi_{0}>0, d \phi / d t>$ 0 for counter-clockwise arcs (as seen from above) and $\delta=\delta_{0}>0, \phi(t)-\phi_{0}<0, d \phi / d t<0$ for clockwise arcs. In addition, the angular range is increased by $\Delta \phi$ with each arc, i.e for the $k$-th $\operatorname{arc}(k=1,2, \ldots)$ we use $\phi_{0, k}<\phi<\phi_{0, k+1}=\phi_{0, k}+k \Delta \phi$ for counter-clockwise arcs and $\phi_{0, k}>\phi>\phi_{0, k+1}=\phi_{0, k}-k \Delta \phi$ for clockwise arcs, and the parameter $r_{0}$ is set to the final value of $r$ in the previous arc.

Paths for $\Delta \phi=30^{\circ}, \phi_{0,1}=0^{\circ}$ and different values of $\delta_{0}$ and are shown in Fig. 3. Note that for $\delta_{0}<90^{\circ}$ the agent will get closer to the nest with time. Thus, while the absolute value of the flight direction angle $|\delta|$ can vary during an arc as observed in real wasps, it has, on average, to be in a quite limited range of about 90 to 100 degrees (see Fig. 1 c,d).

In order to extend the simple model to three dimensions, we make use of the fact that the nest is approximately held at a constant elevation angle $\varepsilon_{\mathrm{H}}$ close to $-45^{\circ}$ (see Fig. $1 \mathrm{c}, \mathrm{f}$ ). For the $z$-coordinate of the agent we then have

$z=r \tan \left|\varepsilon_{\mathrm{H}}\right|$

or simply $z=r$ for $\varepsilon_{\mathrm{H}}=-45^{\circ}$.

2.2 Head-centered model including nest-angle dependent saccades

The model presented in the previous section does not consider the orientation of the insect. Intuitively one might assume the insect's gaze direction to be aligned with flight direction, which would mean that insects would see their nest at angle $\delta$, i.e. in the lateral field of view. However, wasps keep the nest in the frontal visual field during learning flights (see Fig. 1e) so that most of the time the head is actually oriented more towards the nest than in flight direction.

From the viewpoint of a wasp (see Fig. 4), an arc with constant flight direction angle $\delta$ could be achieved by keeping both the nest and the direction of flight at fixed angles in their visual field, i.e. keep angles $\alpha_{\mathrm{H}}$ (the retinal azimuth angle, where the nest is seen) and $\delta_{\mathrm{H}}$ (the retinal flight direction angle) fixed (index ' $\mathrm{H}$ ' indicates the head-centered reference frame) so that $\delta=\delta_{\mathrm{H}}-\alpha_{\mathrm{H}}$ is constant for each arc. Since head orientation described by gaze angle is $\gamma=\phi-\alpha_{\mathrm{H}}$, head orientation would change constantly (with $\phi$ ) during the learning flight. However, learning and return flights in ground-nesting wasps, honeybees and bumblebees have a saccadic organization: rapid head turns ("head saccades") alternate with short flight segments where head orientation is kept virtually constant (Zeil 1993a; Zeil et al. 2007; Boeddeker et al. 2010; Stürzl et al. 2016). Note that we measure gaze angle $\gamma$ with respect to the $-y$-direction.

As a first extension of the initial model we assume that between saccades, i.e. during the intersaccadic interval, gaze angle $\gamma$ is constant, leading to a polygonal approximation of the logarithmic spiral. Head orientation is changed due to saccades of amplitude $\left|\Delta \gamma_{\text {sac }}\right|$ that are triggered whenever nest angle $\alpha_{\mathrm{H}}$ exceeds threshold value $\alpha_{\text {thresh }}$. After the saccade the nest angle is therefore $\alpha_{\mathrm{H}}=\alpha_{\text {thresh }}-\Delta \gamma_{\mathrm{sac}}$ and the absolute value of the gaze angle has increased by $\left|\Delta \gamma_{\text {sac }}\right|$. Note that for counter-clockwise arcs the simulated wasp will turn counter-clockwise during saccades, i.e. $\Delta \gamma_{\mathrm{sac}}>0$, as soon as $\alpha_{\mathrm{H}}>\alpha_{\text {thresh }}=\left|\alpha_{\text {thresh }}\right|>0$ but will perform clockwise saccades $\Delta \gamma_{\mathrm{sac}}<0$, whenever $\alpha_{\mathrm{H}}<\alpha_{\text {thresh }}=$ $-\left|\alpha_{\text {thresh }}\right|<0$ for clockwise arcs.

The initial nest angle is $\alpha(t=0)=0^{\circ}$, corresponding to gaze angle $\gamma(t=0)=0^{\circ}\left(\phi(t=0)=0^{\circ}\right)$. An arc is terminated and a new arc is initiated as soon as the total change of gaze angle during one arc, $\left|\Delta \gamma_{\text {tot }}\right|$, reaches a threshold $\left|\gamma_{\text {thresh }}\right|$ that increases by $\left|\Delta \gamma_{\text {thresh }}\right|$ with each arc. In our model, this is checked whenever the nest angle $\alpha_{\mathrm{H}}$ reaches $\alpha_{\text {thresh }}$, triggering a head saccade. If, after the saccade, the threshold is reached $\left(\left|\Delta \gamma_{\text {tot }}\right|>\left|\gamma_{\text {thresh }}\right|\right)$, a new arc in the opposite direction is started immediately. Similar to the model in the previous section, the change in flight direction is realized by flipping the sign of the flight direction angle. However, we now change, instead of the nest-centered angle $\delta$, the head-centered flight direction angle $\delta_{\mathrm{H}}$, i.e. $\delta_{\mathrm{H}}=\mp \delta_{0, \mathrm{H}}$, where the '-' sign is for counter-clockwise arcs, ' + ' for clockwise arcs, and $\delta_{0, \mathrm{H}}>0$ is the amplitude of the flight direction angle in the head-centered coordinate system. During the first intersaccadic interval at the beginning of each arc, the nest angle $\alpha_{\mathrm{H}}$ changes sign, i.e. the nest moves from one side to the other in the wasp's visual field.

An example showing the time-course of the relevant angles and the resulting path is presented in Fig. 5. With each arc $\left|\gamma_{\text {thresh }}\right|$ was increased by $\left|\Delta \gamma_{\text {thresh }}\right|=$ $30^{\circ}$. Note that for a short period before and after change of flight direction the path follows the same straight line but in opposite direction for the chosen parameter values $\left(\delta_{0, \mathrm{H}}=80^{\circ}, \Delta \gamma_{\mathrm{sac}}= \pm 20^{\circ}\right)$ because $\mid \delta_{\mathrm{H}}-\left(\Delta \gamma_{\mathrm{sac}}+\right.$ $\left.\delta_{\mathrm{H}}^{\prime}\right)\left|=2 \delta_{0, \mathrm{H}}+\right| \Delta \gamma_{\mathrm{sac}} \mid=180^{\circ}$, where $\delta_{\mathrm{H}}$ and $\delta_{\mathrm{H}}^{\prime}=-\delta_{\mathrm{H}}$ are the flight angles (in head coordinates) before and after change of direction.

Average nest-centered flight angle is $\langle|\delta|\rangle=\left\langle\left|\alpha_{\mathrm{H}}\right|\right\rangle+$ $\left|\delta_{\mathrm{H}}\right| \approx \frac{\left|\alpha_{\text {thresh }}\right|+\left(\left|\alpha_{\text {thresh }}\right|-\left|\Delta \gamma_{\mathrm{sac}}\right|\right)}{2}+\delta_{0, \mathrm{H}}$. For the chosen parameter values this gives $\langle|\delta|\rangle=\frac{25^{\circ}+5^{\circ}}{2}+80^{\circ}=95^{\circ}$. Thus, Fig. 5 b corresponds to the path of the continuous 

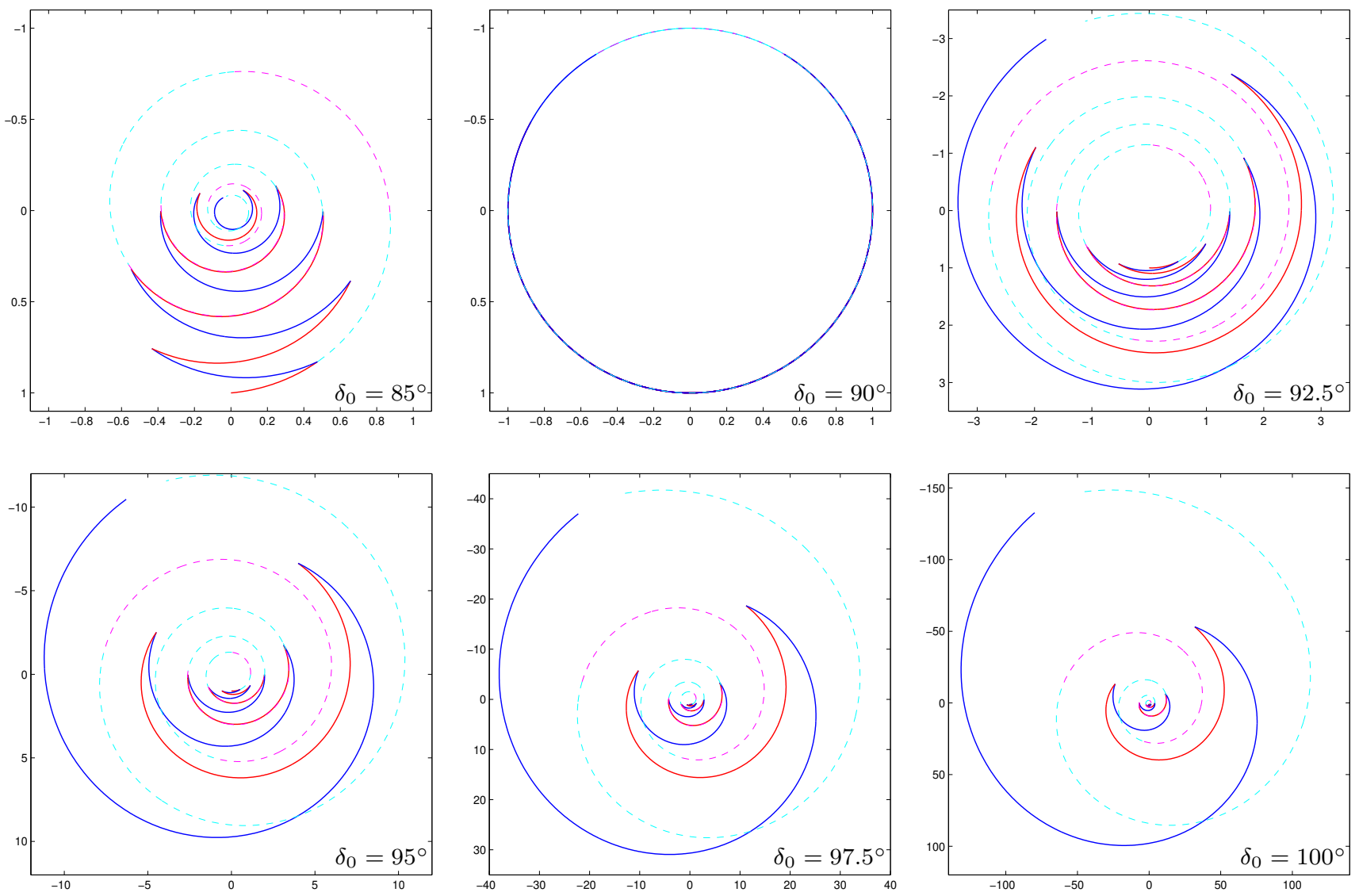

Fig. 3 Paths for different $\delta_{0}$ values and $r_{0}=1$. From top left to bottom right: $\delta_{0}=85^{\circ}, 90^{\circ}, 92.5^{\circ}, 95^{\circ}, 97.5^{\circ}, 100^{\circ}$. For each arc the angular range of $\phi$ is increased by $\Delta \phi=30^{\circ}$. Counter-clockwise arcs (with $\delta=-\delta_{0}$ ) are shown in red, clockwise arcs $\left(\delta=\delta_{0}\right)$ in blue. Dashed curves show paths if flight direction would not change but continued in the same direction. Note that the agent moves towards nest for $\delta_{0}=85^{\circ}<90^{\circ}$.

model for $\delta_{0}=95^{\circ}$, shown in the lower left plot in Fig. 3 .

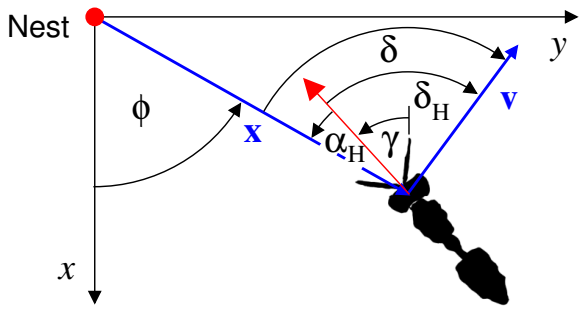

Fig. 4 Wasp and nest centered coordinates and angles. The gaze angle $\gamma$, measured with respect to the negative $y$-axis, describes the head orientation in nest-centered reference system. Angle $\alpha_{\mathrm{H}}$ is the azimuth angle, where the nest is seen in the reference frame of the wasp. As illustrated, the relation between bearing angle $\phi$, nest azimuth angle $\alpha_{\mathrm{H}}$ and gaze angle $\gamma$ is $\phi=\gamma+\alpha_{\mathrm{H}}$. The wasp-centered flight direction angle $\delta_{\mathrm{H}}$ is related to $\delta$, the flight direction angle relative to nest, according to $-\delta=-\delta_{\mathrm{H}}+\alpha_{\mathrm{H}}$. Note that $\delta, \delta_{\mathrm{H}}<0$ as drawn (counter-clockwise arc).
2.3 Visual servoing model based on retinal nest position

We now extend the simple head-centered model of the previous section to a visual servoing model and show its functionality in closed-loop simulation with visual input consisting of rendered panoramic images using 3D reconstructions of wasp nesting sites (see Stürzl et al. 2015). In addition to the constant flight angle and the two threshold-based rules already described, namely

1) keep head centered flight direction $\delta_{\mathrm{H}}$ fixed during one arc, change sign with the beginning of each arc,

2) start new arc in the opposite direction if total change of gaze angle $\left|\Delta \gamma_{\text {tot }}\right|$ along an arc exceeds $\left|\gamma_{\text {thresh }}\right|$, increase $\left|\gamma_{\text {thresh }}\right|$ by $\left|\Delta \gamma_{\text {thresh }}\right|$ with each arc,

3) keep nest azimuth angle within the frontal visual field by initiating rapid turns of the head with amplitude $\Delta \gamma_{\text {sac }}$, whenever nest azimuth angle $\alpha_{\mathrm{H}}(t)$ reaches $\alpha_{\text {thresh }}$,

we propose two simple continuous control rules. They are, motivated by observations and experiments (Zeil 

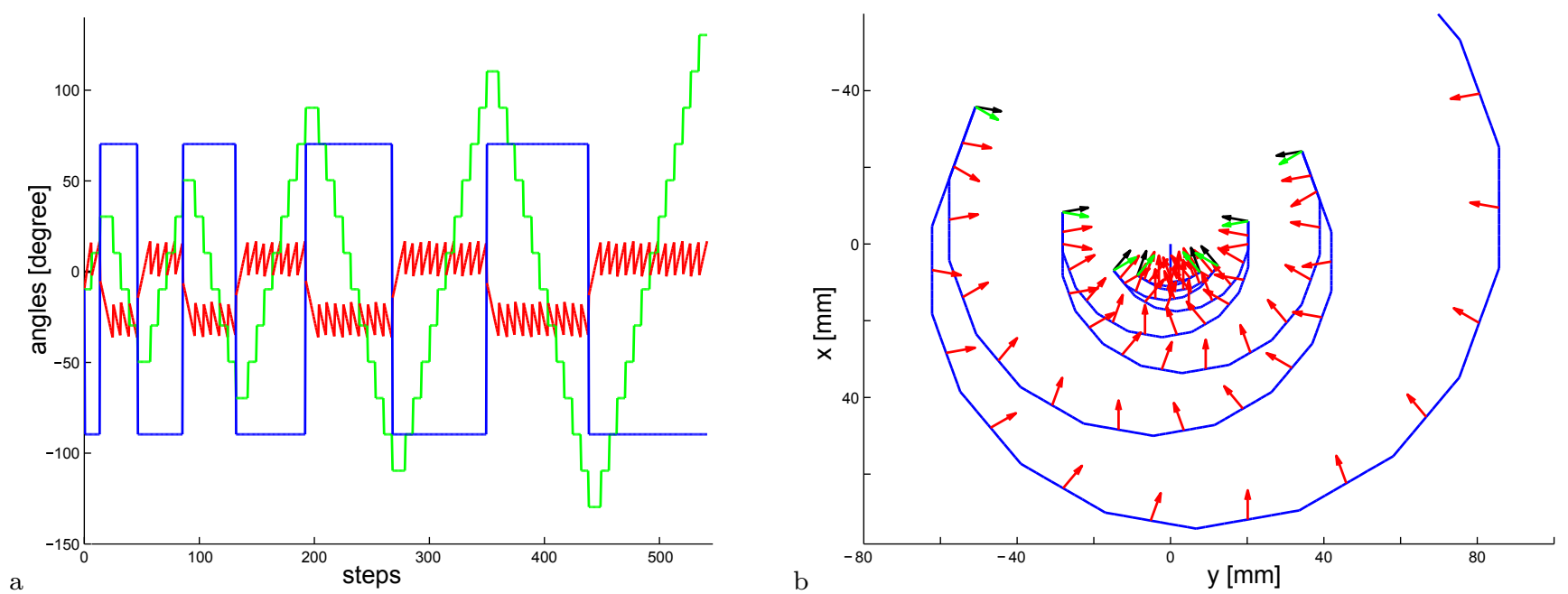

Fig. 5 Head-centered model with nest angle dependent saccades generating $n=9 \operatorname{arcs}$. Parameter values are $\left|\alpha_{\text {thresh }}\right|=25^{\circ}$ (nest angle threshold), $\left|\Delta \gamma_{\mathrm{sac}}\right|=20^{\circ}$ (saccade amplitude), $\delta_{0, \mathrm{H}}=80^{\circ}$ (flight angle amplitude) and $\left|\gamma_{\mathrm{thresh}, k}\right|=k \Delta \gamma$, where $\Delta \gamma=30^{\circ}, k=1,2, . ., n$. Initial distance from nest is $r_{0}=10 \mathrm{~mm}$ (dashed blue line). a) Time course of angles: Head orientation/gaze $\gamma$ (green), nest azimuth in head-centered reference system $\alpha_{\mathrm{H}}$ (red), flight angle in head coordinates $\delta_{\mathrm{H}}$ (blue). b) Top-down view of path (blue): Head orientation is indicated by small red arrows at the central position of each intersaccadic interval. The final saccade at the end of each arc is illustrated by black and green arrows. The new gaze direction (green arrow) is kept until the nest azimuth angle reaches threshold on the other side, making the first intersaccadic interval longer than the following intervals.

1993a,b; Zeil et al. 1996; Zeil 1997; Zeil et al. 2007; Stürzl et al. 2016), based on the angular velocity and retinal position of the nest:

4) change horizontal speed so that (horizontal) retinal velocity of nest is nearly constant $\left(\dot{\alpha}_{\mathrm{H}}(t) \approx \dot{\alpha}_{\mathrm{H}}^{*}\right)$ between saccades:

$$
\frac{d}{d t} v_{x y}(t)=K_{P, v}\left(\dot{\alpha}_{\mathrm{H}}^{*}-\dot{\alpha}_{\mathrm{H}}(t)\right)
$$

5) change height $z(t)$ in order to keep nest elevation angle nearly constant $\left(\varepsilon_{\mathrm{H}}(t) \approx \varepsilon_{\mathrm{H}}^{*}\right)$ :

$$
\frac{d}{d t} z(t)=-K_{P, z}\left(\varepsilon_{\mathrm{H}}^{*}-\varepsilon_{\mathrm{H}}(t)\right) .
$$

Since horizontal speed as well as height increases with growing distance to the nest during a learning flight (Zeil et al. 1996), the coefficients (or gains) of the simple proportional controllers, $K_{P, v}$ and $K_{P, z}$ should also increase with time. In our model they are increased with each new arc, but kept constant during an arc, i.e. they are proportional to the arc number $k=1,2, \ldots: K_{P, v}=$ $k K_{P, v}^{0}, K_{P, z}=k K_{P, z}^{0}$. Alternatively, they could be increased proportionally with time. Also feed-forward terms, i.e. horizontal acceleration in (4) and vertical speed in (5) could be added.

Note that the control rules do not contain any measurements of position, distance to ground or flight speed. The resulting path is scaled by the initial distance to the nest (after leaving the nest and turning towards the nest), described by parameter $r_{0}$.
For the simulation results presented here, we use $K_{P, v}^{0}=K_{P, z}^{0}=10^{-3} \frac{\mathrm{m}}{{ }_{\mathrm{s}}}$. As set points we choose $\varepsilon_{\mathrm{H}}^{*}=$ $-45^{\circ}, \dot{\alpha}_{\mathrm{H}}^{*}=200^{\circ} \mathrm{s}$. Initial values are $r_{0}=0.01 \mathrm{~m}, z_{0}=$ $r_{0} \tan \left|\varepsilon_{\mathrm{H}}^{*}\right|=r_{0}, v_{x y, 0}=r_{0} \dot{\alpha}_{\mathrm{H}}^{*} \approx 0.035 \frac{\mathrm{m}}{\mathrm{s}}$ and $\gamma_{0}=0$. Parameters are set to $\left|\Delta \gamma_{\mathrm{sac}}\right|=20^{\circ},\left|\alpha_{\text {thresh }}\right|=27.5^{\circ}$ and $\delta_{0, \mathrm{H}}=80^{\circ}$. Compared to the previous section we increased $\left|\alpha_{\text {thresh }}\right|$ slightly so that the average nest centered flight angle is now $\langle|\delta|\rangle \approx \frac{\left|\alpha_{\text {thresh }}\right|+\left(\left|\alpha_{\text {thresh }}\right|-\left|\Delta \gamma_{\text {sac }}\right|\right)}{2}$ $+\delta_{0, \mathrm{H}}=97.5^{\circ}$, which would lead to somewhat larger distances between arcs (see Fig. 3). However, this is approximately compensated for by additional loops due to the "continuous change of flight angle at the end of arcs" described below.

Continuous change of flight angle at the end of arcs. In the learning flights of real wasps, flight direction does not change instantly but continuously at the end of an arc / beginning of a new arc. We use the heuristic function $\delta_{\mathrm{H}}(\tilde{t})=\delta_{0, \mathrm{H}}(1-2 f(\tilde{t}))$ with $f(\tilde{t})=6 \tilde{t}^{5}-$ $15 \tilde{t}^{4}+10 \tilde{t}^{3} \in[0,1], \tilde{t} \in[0,1]$, for describing the (continuous) transition of the flight angle from $\delta_{0, \mathrm{H}}$ to $-\delta_{0, \mathrm{H}}$ (and similarly from $-\delta_{0, \mathrm{H}}$ to $\delta_{0, \mathrm{H}}$ ). We assume its duration to be proportional to horizontal flight speed, i.e. $T_{\text {trans }}=c_{\text {trans }} v_{x y}$, and therefore $\tilde{t}=\left(t-t_{0}\right) / T_{\text {trans }}$, $\tilde{t} \in\left[t_{0}, t_{0}+T_{\text {trans }}\right]$, where $t_{0}$ is the start of the flight direction change, while flight speed $v$ (as well as head orientation) is kept constant. For the examples shown in the following we set $c_{\text {trans }}=0.5 \frac{\mathrm{s}^{2}}{\mathrm{~m}}$.

During change of flight direction, the wasp will move somewhat closer to the nest in the $x-y$ plane, which will 
cause a slight drop in $z$ since control rule (5) tries to keep elevation constant.

\section{Visual nest tracking}

We also implemented visual tracking of the nest position (with continuously updated template), similar to (Samet et al. 2014), that allows us to generate artificial learning flights in closed-loop in 3D models of real wasp environments. The tracked nest position provides the parameters, essential for the visual servoing, $\alpha_{\mathrm{H}}(t)$, $\varepsilon(t)$ and $\dot{\alpha}_{\mathrm{H}} \approx \frac{\alpha_{\mathrm{H}}(t)-\alpha_{\mathrm{H}}(t-\Delta t)}{\Delta t}$, where $\Delta t$ is the time between two consecutive frames (the time discretization in the simulation).

As shown in Fig. 6, a rectangular area is used as a template. Initially, when tracking starts, the template consists of a blurred dark circle resembling the entrance of the nest. Subsequently, the template is updated regularly with the best matching image region, that is the image region centered around pixel position $x^{*}, y^{*}$ which has the highest similarity to the current template. Full-spherical images are rendered in equirectangular projection with resolution of $2^{\circ}$ per pixel from $3 \mathrm{D}$ models that are reconstructions of natural wasp nesting sites (Stürzl et al. 2015). We use the sum of absolute pixel value differences (SAD) as image similarity metric. The minimum of the $\mathrm{SAD}$ values over the search region defines the estimate of the retinal nest position, i.e.

$\left(x_{t}^{*}, y_{t}^{*}\right)=\arg \min _{(x, y) \in U_{t-1}} \operatorname{SAD}(x, y)$,

where $U_{t-1}=U\left(x_{t-1}^{*}, y_{t-1}^{*}\right)$ is the search region centered at the best matching position in the previous time step. In our implementation, the template is updated every $100 \mathrm{~ms}$. In case a saccade has been initiated after the previous tracking step, the search region is shifted accordingly, i.e. by $\left|\Delta \gamma_{\mathrm{sac}}\right| /$ resolution $=20^{\circ} / 2 \% \mathrm{px}=$ $10 \mathrm{px}$ to the left or to the right depending on the saccade being clockwise or counter-clockwise.

Continuous update of the template allows to track the nest region over the whole learning flight, but will also cause drifts, because the deviation of the estimated from the true nest position usually increases with time. Fortunately, high accuracy is only needed close to the nest because the homing method currently proposed for ground nesting wasps is based on the assumption that insects store views during their learning flights that are tagged with the information 'nest left' or 'nest right', which then provides navigational instructions 'move left' or 'move right' during the return to the nest (Stürzl et al. 2016). The exact nest angle is therefore not important.

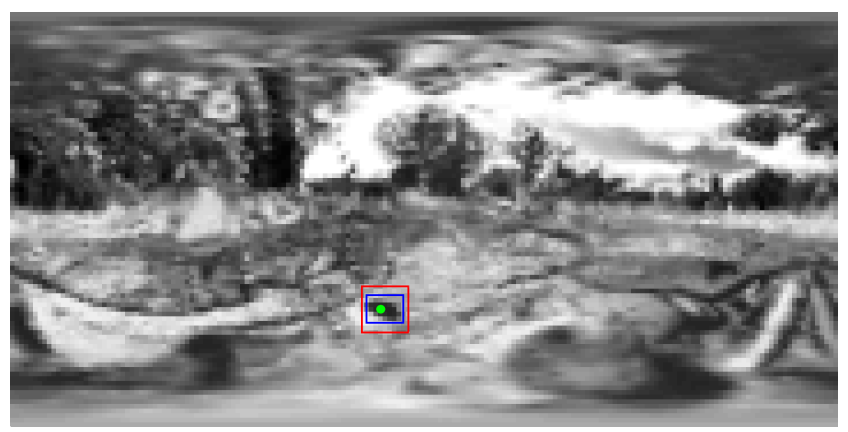

Fig. 6 Template tracking of the nest region in rendered panoramic images. The best match within the search region (blue rectangle) is used as the estimated retinal nest position. The true nest position is highlighted by a green dot. The red rectangle depicts the image region that matches the current template best. Images are rendered at a rate of $60 \mathrm{~Hz}$. Every 100 msec (of simulation time), i.e. every 6th rendered image, the best matching image region replaces the template ("template update").

Results for different nest sites, with images rendered from 3D models, are shown in Fig. 7. The 3D-models of environment 1, located at the Mount Majura Nature Reserve, Canberra, Australia, with detailed reconstruction of a single nest location, and of environment 2, located at the Australian National University, with two nest locations were generated from scans and camera images (see (Stürzl et al. 2016) for details). As can be seen in Fig. 7, nest tracking may lead to deviations of the estimated from the true retinal nest position, which can result in variations in the learning flight paths.

Instead of this simple approach a more sophisticated tracking method could be used that may improve this situation. However, how nest tracking is realized in real wasps is unclear and thus any improvements would, at least currently, not be supported by biological evidence. Also the visual resolution of real insects could be taken into account. Preliminary tests of nest tracking using "bee-eye views", rendered according to the model described in (Stürzl et al. 2010), showed comparable performance (Samet et al. 2014).

\section{Close-range visual homing based on views memorized during a generated learning flight}

In order to test whether the views recorded during learning flights generated by our model are sufficient for the return to the nest we applied the model for visual homing described in (Stürzl et al. 2016). It basically assumes that wasps store views during their learning flights tagged with the information 'nest left' or 'nest right'. During return they then move according to nest direction of the stored view that is most similar to the current view. For the simulated return flights 

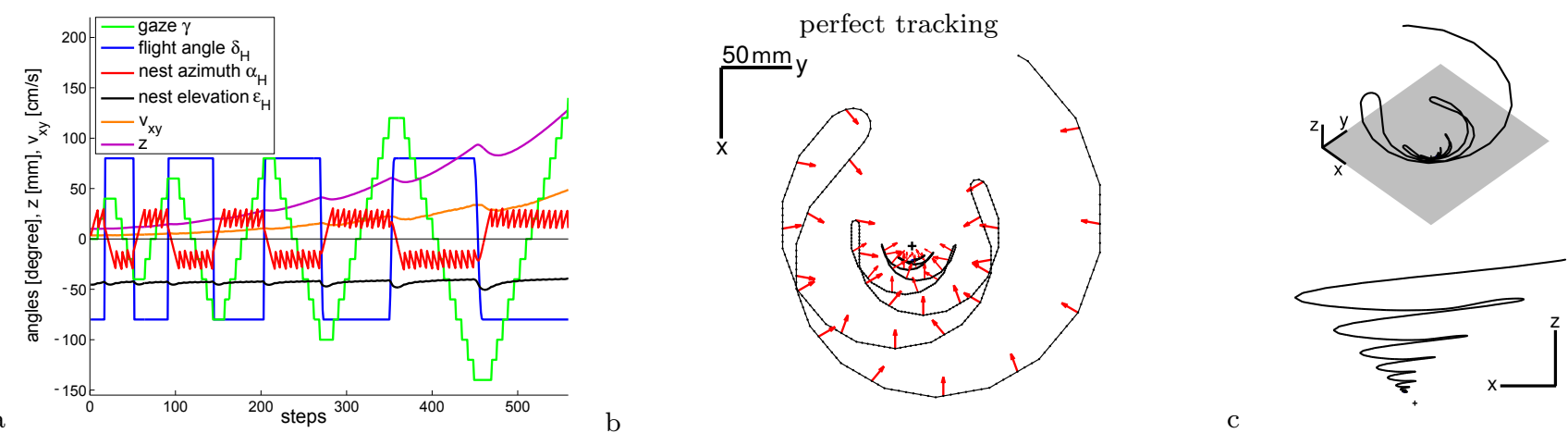

$\mathrm{b}$

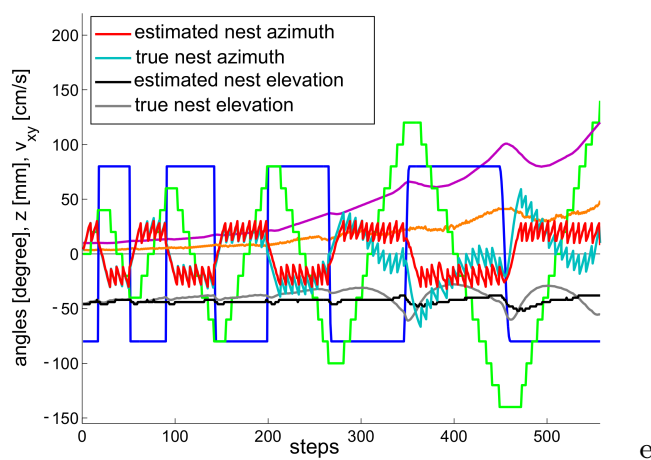

environment 1
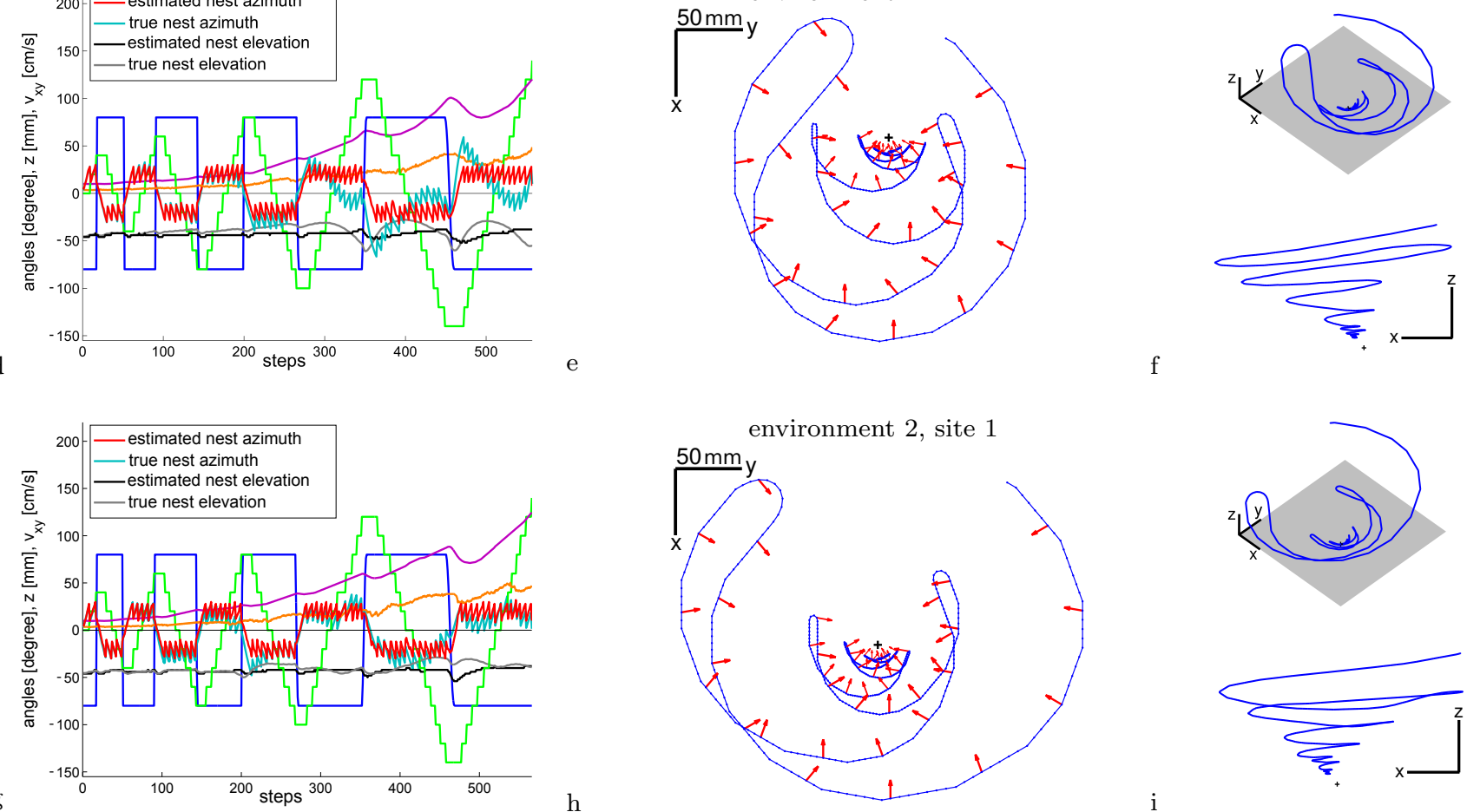

environment 2 , site 1
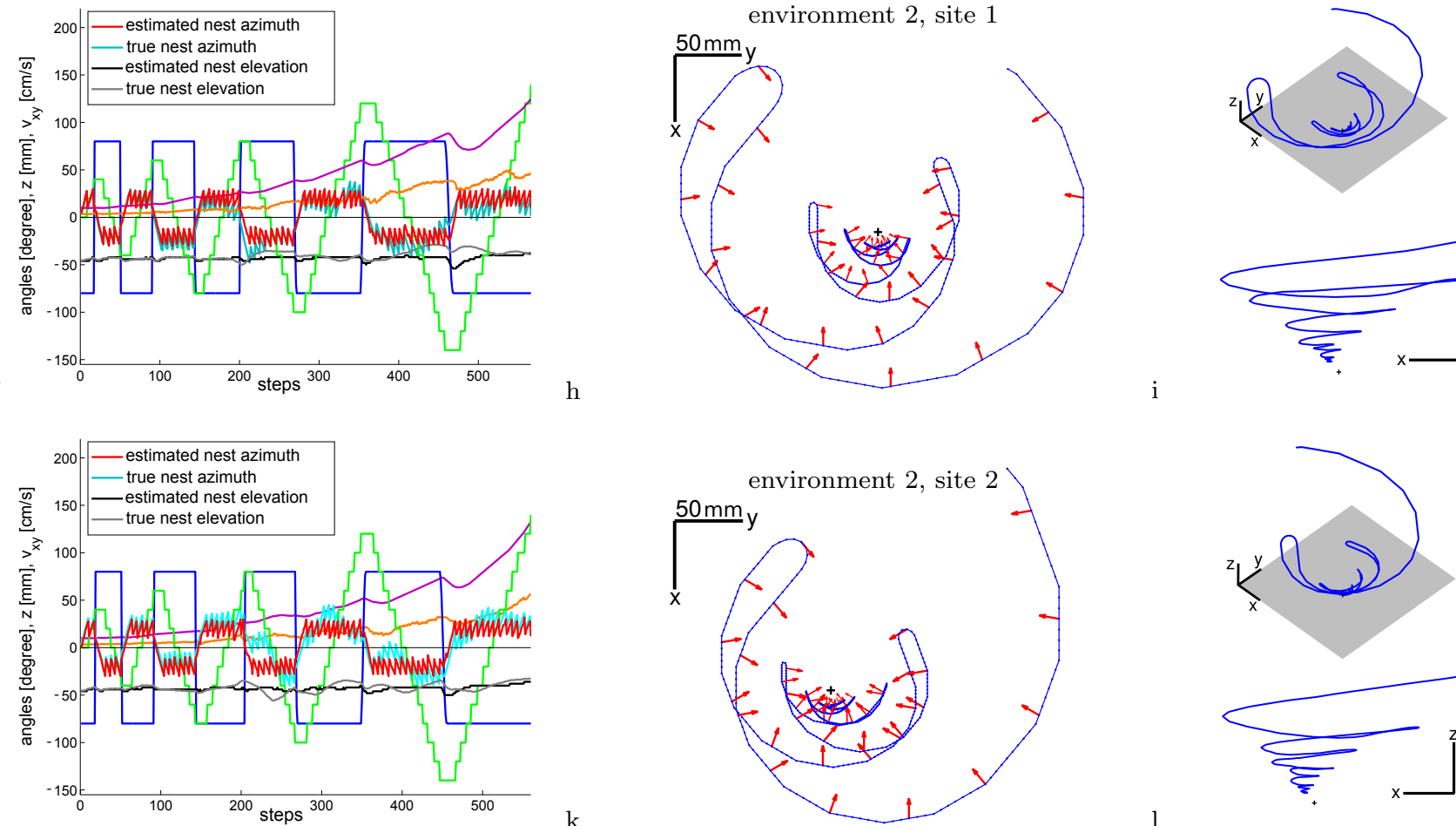

$\mathrm{h}$
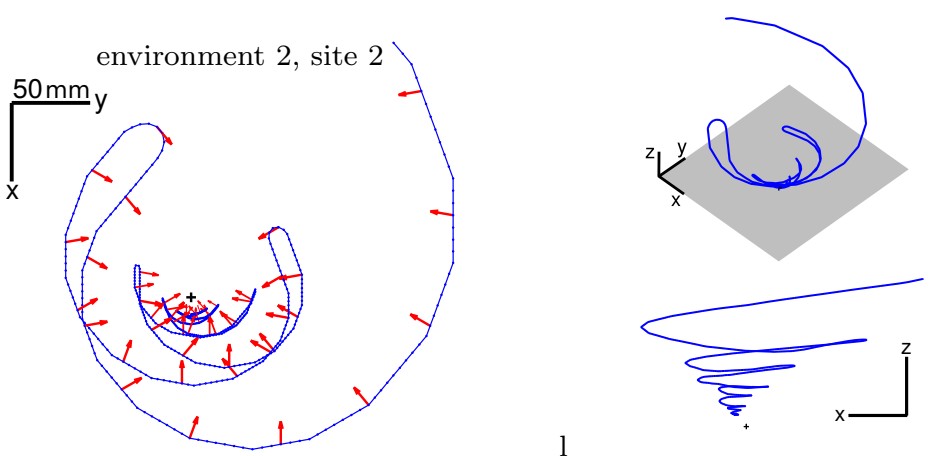

Fig. 7 Results for three different environments/nesting sites. In the left column, the following parameters are plotted over simulation steps: head angle $\gamma$ (green), head centered flight angle $\delta_{\mathrm{H}}$ (blue), the estimated (from tracking) / true nest azimuth $\alpha_{\mathrm{H}}$ (red / light blue) and elevation angle $\varepsilon$ (black / gray), height $z$ in mm (magenta) and (horizontal) flight speed $v_{x y}$ in $\mathrm{cm} / \mathrm{s}$ (orange). The middle column shows top views of the generated learning flights with head orientations depicted by red arrows every 10th simulation step. The right column contains side views of learning flights from different viewing directions. a)-c) Time course of angles and path in case of perfect tracking, i.e. estimated nest azimuth and elevation angle have true values. d)-1) Results from template-based nest tracking using images rendered from 3D models of nesting sites. Differences of the resulting paths are due to "tracking errors", i.e. deviations of the true from the estimated nest direction (azimuth and elevation) and are dependent on the environment (depth structure and texture). Parameter values: $r_{0}=10 \mathrm{~mm},\left|\Delta \gamma_{\mathrm{sac}}\right|=20^{\circ}$, $\delta_{0, \mathrm{H}}=80^{\circ},\left|\alpha_{\text {thresh }}\right|=27.5^{\circ},\left|\Delta \gamma_{\text {thresh }}\right|=30^{\circ}$. 

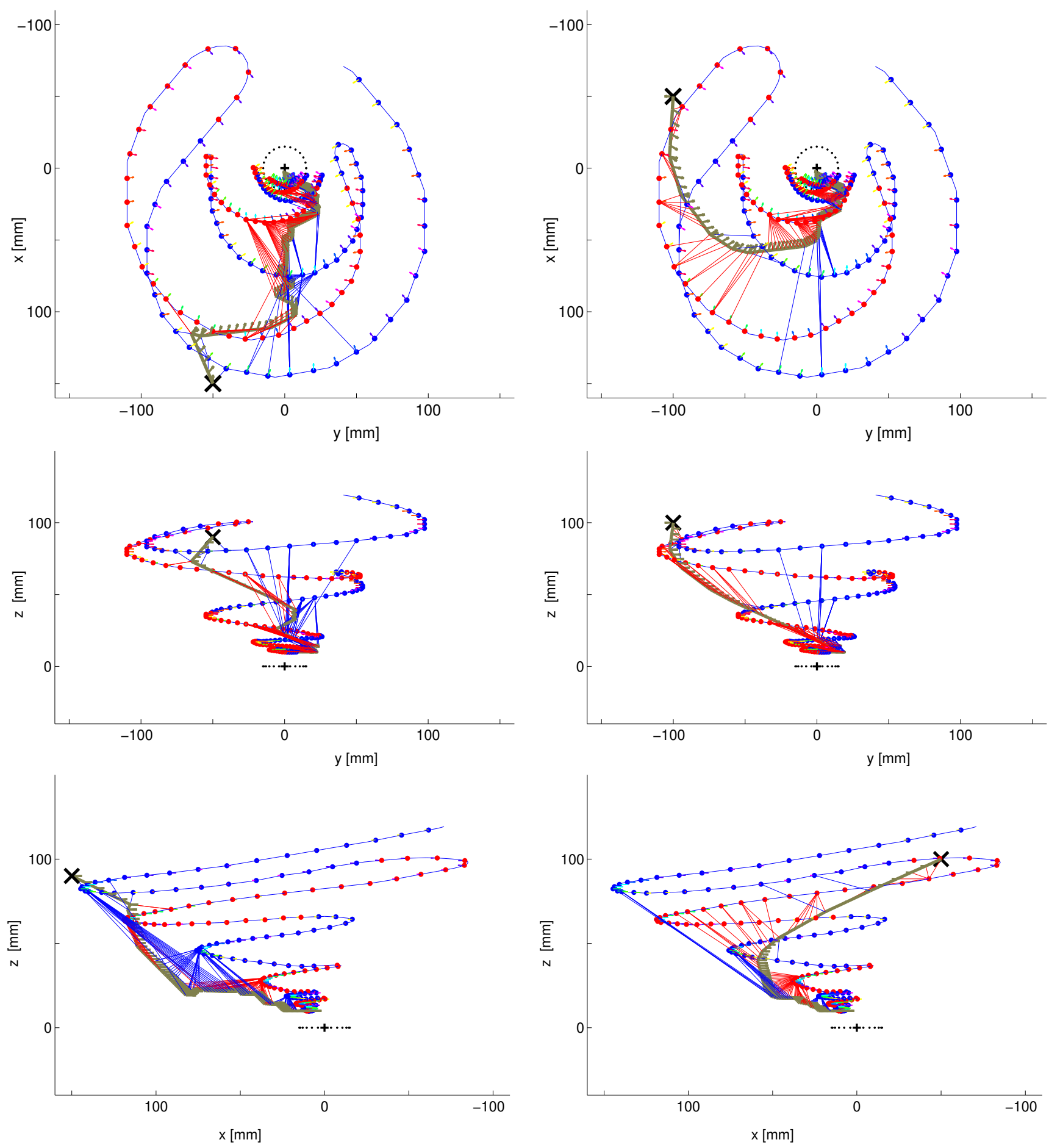

Fig. 8 Simulated return flights (brown curves with arrows indicating head orientations, black ' $X$ ' highlight start positions) guided by views of the artificial learning flight in environment 1, see Fig. 7 d-f. Every third image rendered during the learning flight was stored as a view for guiding the return (185 views in total). Left column: Starting at (150, -50, 90) mm. Right column: Start position $(-50,-100,100) \mathrm{mm}$. Top row: top-down views onto $x-y$ plane, middle row: side view on $x-z$ plane, bottom row: side view on $y-z$ plane. Blue or red lines connecting return flight positions to positions of the learning flight indicate the position of the learning flight view used for guiding the simulated wasp to the left or to the right depending on whether the nest was on the left or the right side during learning. Dotted black circle illustrate the "nest range" inside which it is assumed that nest the entrance can be detected and homing stops once the entrance has moved from one side to the other in the visual field. 
shown in Fig. 8, the model was implemented as described in the supplementary material of (Stürzl et al. 2016) with the difference that a return flight terminates if the simulated wasp is inside the "nest range", defined by $\sqrt{x^{2}+y^{2}}<15 \mathrm{~mm}$, and the retinal position of the nest entrance has moved from one side of the visual field to the other, i.e. nest azimuth angle has changed sign. Inside the nest range we assume that the nest entrance can be detected. As shown based on two examples in Fig. 8, views with nest position tags, memorized during a learning flight generated by our model, can guide the simulated wasp towards the nest. Note that these tags depend on the tracking results, i.e. whenever the nest was estimated to be on the left side the corresponding views will guide the simulated wasp to the left during homing, even if the true retinal nest position was on the other side. While such errors would make finding the nest very difficult if nest tags are wrong for views very close to the nest, they are much less problematic for views further away, as they would lead to deviations from the optimal path that can be corrected by views closer to the nest.

\section{Discussion}

We presented a model for visually controlled learning flights that produces paths similar to those of groundnesting wasps. We also showed that views stored while moving along the generated path can be used for homing.

We do not claim that these are the exact control rules used by learning insects. Our aim was to show that a small set of biologically plausible rules can result in paths similar to the learning flights observed in groundnesting wasps. Our results indicate that similar control rules could be implemented on a small flying system or MAV (micro aerial vehicle) with limited computing power.

In the following we discuss a number of limitations and possible extensions of the model.

Estimating flight direction angle. In our model we have simply assumed that the head-centered flight direction angle $\delta_{\mathrm{H}}$ is known and can be kept constant with high accuracy. In flying insects, optic flow (see e.g. Koenderink and van Doorn 1987) is likely to be the main source of information for estimating and controlling $\delta_{\mathrm{H}}$. Insect-inspired models for estimating flight direction from optic flow exist (Franz et al. 2004; Strübbe et al. 2015) and could be integrated into the simulation.

Complementing tracking with visual odometry. The central input to our visual servoing model is the retinal nest position estimated by tracking. In addition, visual odometry (Srinivasan et al. 2000), or path integration, based on optic flow and other sensors, e.g. a celestial compass, could be used to estimate the direction and the distance of the nest.

Estimating total change of gaze angle. According to the model, arcs get larger with duration of the learning flight because distance to nest increases with time and angular range increases with number of arcs due to rule (2), which is based on the total change of gaze angle along an arc, $\Delta \gamma_{\text {tot }}$. The value of $\Delta \gamma_{\text {tot }}$ could be estimated in a number of ways: using some kind of compass, the dissimilarity of the current view to views on previous arcs (Stürzl et al. 2016), the duration of an arc (that should be longer than previous one), or by counting saccades. As our model assumes that all head rotations are due to saccades of fixed amplitude, the change in head orientation during one arc is $\left|\Delta \gamma_{\text {tot }}\right|=n_{\text {sac }}\left|\Delta \alpha_{\text {sac }}\right|$, where $n_{\text {sac }}$ is the number of saccades that usually increases with every arc. Deviations from this rule that sometimes can be observed in real flights, i.e. non-increasing range of head orientations for one arc compared to the previous arc, could then be explained by some kind of "counting error". Of course, other reasons are possible, e.g. certain orientations might not be important as the nest will not be approached from some directions due to the specific structure of the scene or prevailing wind direction. In addition, the amplitudes of saccades in real learning flights varies slightly, with a median of $22^{\circ}$ and interquartile range of $11^{\circ}$ (Stürzl et al. 2016).

Alternative control of saccades. In our model we assume that nest azimuth position is constantly monitored and head saccades of constant amplitude are initiated whenever a position error threshold is reached. The saccade amplitude variation of real learning flights could be easily incorporated by generating random amplitudes according to a defined distribution.

A slight modification of this scheme, motivated by Zeil (1997), who found that a rotating drum (eliciting optomotor responses) did not change frequency of saccades $^{1}$, is that nest position could be checked regularly (interval $\Delta T$ ) and a saccade triggered with amplitude proportional to the deviation of the estimated nest position $\alpha_{\mathrm{H}}$ from set point $\alpha_{\mathrm{H}}^{*}$, i.e.

$$
\Delta \gamma_{\mathrm{sac}}(t=k \Delta T) \propto \alpha_{\mathrm{H}}(t)-\alpha_{\mathrm{H}}^{*},
$$

1 Note that Zeil (1997) actually reports rapid changes in body orientation (body saccades). Body saccades accompany head saccades which has been observed not only in wasps (Stürzl et al. 2016), but also in bumble bees (Riabinina et al. 2014; Boeddeker et al. 2015) and honeybees (Boeddeker et al. 2010). 
if $\left|\alpha_{\mathrm{H}}(t)-\alpha_{\mathrm{H}}^{*}\right|$ exceeds some minimum value.

Alternative control of flight speed. Instead of the angular velocity of the retinal nest position $\dot{\alpha_{\mathrm{H}}}$, the ventral optical flow $\mathrm{OF}_{\mathrm{v}}$, as suggested in Zeil (1997) and Linander et al. (2018), or a combination of both, could serve as "process variable" for controlling horizontal speed during learning flights as well. Assuming even ground, a constant elevation angle $\varepsilon_{\mathrm{H}} \approx \varepsilon_{\mathrm{H}}^{*}$ combined with constant horizontal angular velocity $\dot{\alpha}_{\mathrm{H}} \approx \dot{\alpha}_{\mathrm{H}}^{*}$ of the retinal nest position will result in constant ventral optical flow amplitude $\mathrm{OF}_{\mathrm{V}}=\frac{v_{x y}}{z} \approx \frac{r \dot{\alpha}_{\mathrm{H}}^{*}}{r \tan \left|\varepsilon_{\mathrm{H}}\right|}=\frac{\dot{\alpha}_{\mathrm{H}}^{*}}{\tan \left|\varepsilon_{\mathrm{H}}\right|}$. For $\tan \left|\varepsilon_{\mathrm{H}}\right| \approx \tan 45^{\circ}=1$ we find $\mathrm{OF}_{\mathrm{v}} \approx \dot{\alpha}_{\mathrm{H}}^{*}$.

While the average time course of increasing flight speed can be modeled quite well by control laws similar to rule (4), high frequency deviations can be observed in real flights, possibly because both acceleration and deceleration are high at the onset and the end of an intersaccadic interval, respectively.

Including flight dynamics. So far we did not consider the specific movement dynamics of flying insects. In particular one has to take into account that the "flight motor" of wasps is attached to the body while visual feedback is obtained in the retinal or head-centered reference system. For instance, the rapid head saccades will not lead to immediate changes in direction in the flight path. Due to momentum, flight angle relative to gaze will increase shortly after a saccade before approaching its set point.

Variability of learning flights. As in our model all parameters and initial values are kept constant, the only variation in the generated paths is due to different tracking results for different environment models.

In contrast, real flights show quite some variability, within and between species and individuals, which can not be explained by tracking errors only. For instance, flight direction is probably estimated from optical flow, which depends not only on the 3D structure but also on other visual properties of the scene, such as contrast, direction of illumination and shadows, and therefore will contribute to the variability of flights. In addition there are differences in experience, flight style (e.g. hovering abilities), aerodynamics (comparing, for instance, bumble bees with wasps, e.g. Philippides et al. 2013 with Collett 1995, Zeil 1993a), and in wind conditions.

Some deviations from the paths generated by our control model might also be due to learning flights serving additional tasks/purposes or having constraints not considered so far. For example, deviations from rules (4) or (5) due to short phases of strong acceleration observed in real flights, might actually be "on purpose".
Assuming that wasps, similar to fruit flies (Kamikouchi et al. 2009), can sense acceleration, this would enable them in combination with optic flow to estimate flight speed or height above ground (Srinivasan 1993).

During their learning flights homing insects acquire information on the location of a goal relative to the landmark panorama and the flights are crucial for the insects being able to pinpoint the nest on subsequent returns (Tinbergen 1932; Tinbergen and Kruyt 1938; Zeil 1993a,b). This is despite the fact that learning flight paths can be quite variable within and between species (e.g. Collett and Lehrer 1993; Zeil 1993a; Philippides et al. 2013). This was also corroborated by simulations with our visual homing model where views along the learning flights of different wasps resulted in successful guidance back to the nest (Stürzl et al. 2016).

\section{Duration of close-range learning flights and subsequent} learning for far-range homing. The oscillatory "arc to the left, arc to the right" pattern of learning flights usually stops after about 10 arcs or less and within about 10 seconds. As each arc takes longer than the previous one despite the increase of horizontal velocity, the oscillatory flight pattern is not effective for larger distances from the nest. As learning insects fly along arcs of increasing radius, their pivoting velocity tends to stay constant because their flight speed increases with distance from the goal and height above ground (Zeil 1993a; Zeil et al. 1996). There must be a limit to how fast the insects can fly and this maximum speed may cause the initial sections of learning flights, where the insects fly sideways around the goal to end. After this close-range learning flight, a "spiraling" flight towards the sky has been described in honeybees. During this phase that Jander (1997) called "peripheral exploration flight" insects fly, their body aligned with flight direction, in circles of increasing diameters upwards.

Most likely, learning flights with their characteristic structure are mainly for pin-pointing a goal location, i.e. for guiding the close-range homing as described in section 3. The spiral-like flight paths that have been described following the learning flights of honeybees (Jander 1997) could provide cues from positions much higher up in the air, where the catchment volume of views is also much larger than closer to ground (Zeil et al. 2003; Stürzl and Zeil 2007; Murray and Zeil 2017). Therefore, the approach from further away could possibly be guided by increasing image similarity to one of the views along the spiral. Once at or close to the spiral, wasps could "dive" towards the ground and start the close-range visual homing, see Fig. 9. This hypothesis would have to be corroborated by further research, of course, recording flights over larger areas than has 


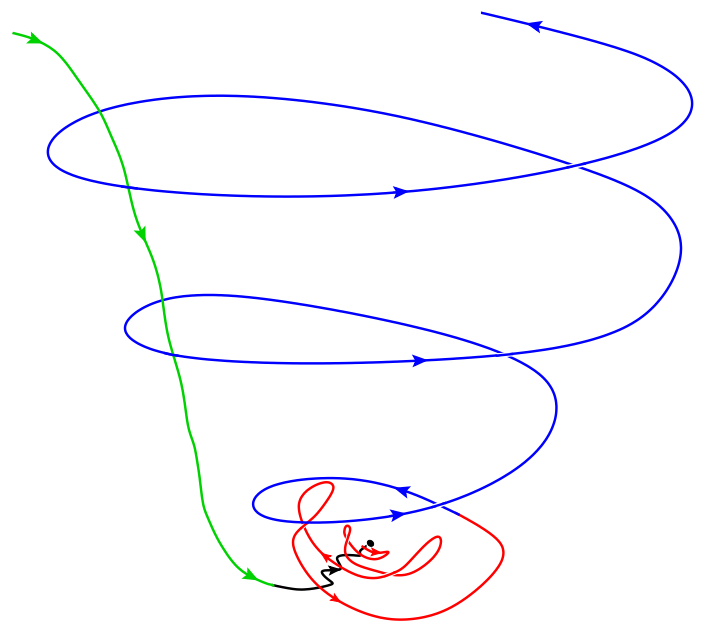

Fig. 9 Sketch of a combined close- and far-range learning and return model. Learning flight: close-range (red), far-range (blue). Return flight: far-range (green), near range (black). Arrows indicate flight direction which can be different from head orientation.

been done so far with cameras - possibly using radar tracking methods (ideally in 3D) or arrays of synchronized cameras.

Acknowledgements We thank Kristin Bussmann for discussions on control theory. The work was supported by the German Science Foundation (PS and WS, DFG grant STU 413/2-1), by an Australian Research Council Discovery Project Grant (JZ and WS, DP150101172) and by the Go8 AustraliaGermany Joint Research Collaboration Scheme (JZ and WS).

\section{Appendix}

\section{Derivation of Eq. (2)}

Substituting $\mathbf{x}=(x, y)^{\top}, x=r \cos \phi, y=r \sin \phi$ in Eq. (1) gives with $\dot{x}=\dot{r} \cos \phi-r \sin \phi \dot{\phi}$ and $\dot{y}=\dot{r} \sin \phi+$ $r \cos \phi \dot{\phi}$,

$$
\begin{aligned}
\left(\begin{array}{c}
\dot{r} \cos \phi-r \sin \phi \dot{\phi} \\
\dot{r} \sin \phi+r \cos \phi \dot{\phi}
\end{array}\right) & =-\lambda\left(\begin{array}{c}
r \cos (\phi+\delta) \\
r \sin (\phi+\delta)
\end{array}\right), \\
\Longleftrightarrow \quad \mathbf{R}(\phi)\left(\begin{array}{c}
\dot{r} \\
r \dot{\phi}
\end{array}\right) & =-\lambda r \mathbf{R}(\phi)\left(\begin{array}{c}
\cos \delta \\
\sin \delta
\end{array}\right), \\
\Longleftrightarrow \quad\left(\begin{array}{c}
\dot{r} \\
r \dot{\phi}
\end{array}\right) & =\lambda r\left(\begin{array}{l}
-\cos \delta \\
-\sin \delta
\end{array}\right),
\end{aligned}
$$

where $\mathbf{R}(\phi)=\left(\begin{array}{rr}\cos \phi & -\sin \phi \\ \sin \phi & \cos \phi\end{array}\right)$. Dividing first by second row (assuming $r, \dot{\phi}, \lambda, \sin \delta \neq 0$ ) and then integrating both sides (assuming $\delta$ constant) results in

$$
\begin{aligned}
& d r / r=\cot \delta d \phi, \\
& \Longleftrightarrow \int_{r_{0}}^{r} \frac{1}{r} d r \stackrel{(r \geq 0)}{=} \ln \left(r / r_{0}\right)=\cot \delta \int_{\phi_{0}}^{\phi} d \phi \\
&=\cot \delta\left(\phi-\phi_{0}\right) .
\end{aligned}
$$

Solving for $r$ finally gives

$r=r_{0} \exp \left[\cot \delta\left(\phi-\phi_{0}\right)\right]$.

\section{References}

Baddeley B, Philippides A, Graham P, Hempel de Ibarra N, Collett T, Husbands P (2009) What can be learnt from analysing insect orientation flights using probabilistic SLAM? Biological Cybernetics 101:169-182

Boeddeker N, Dittmar L, Stürzl W, Egelhaaf M (2010) The fine structure of honeybee head and body movements in a homing task. Proceedings of the Royal Society B: Biological Sciences 277:1899-1906

Boeddeker N, Mertes M, Dittmar L, Egelhaaf M (2015) Bumblebee homing: The fine structure of head turning movements. PLOS ONE 10:e0135020

Brünnert U, Kelber A, Zeil J (1994) Ground-nesting bees determine the location of their nest relative to a landmark by other than angular size cues. Journal of Comparative Physiology A 175:363-369

Collett T (1995) Making learning easy: the acquisition of visual information during the orientation flights of social wasps. Journal of Comparative Physiology A 177:737-747

Collett T, Lehrer M (1993) Looking and learning: a spatial pattern in the orientation flight of the wasp Vespula vulgaris. Proceedings of the Royal Society B: Biological Sciences 252:129-134

Collett TS, Zeil J (2018) Insect learning flights and walks. Current Biology 28:R984-R988

Collett TS, Hempel de Ibarra N, Riabinina O, Philippides A (2013) Coordinating compass-based and nest-based flight directions during bumblebee learning and return flights. Journal of Experimental Biology 216:1105-1113

Dewar AD, Philippides A, Graham P (2014) What is the relationship between visual environment and the form of ant learning-walks? An in silico investigation of insect navigation. Adaptive Behavior 22:163-179

Dittmar L (2011) Static and dynamic snapshots for goal localization in insects? Communicative and Integrative Biology 4:17-20

Dittmar L, Stürzl W, Baird E, Boeddeker N, Egelhaaf M (2010) Goal seeking in honeybees: matching of optic flow snapshots? Journal of Experimental Biology 213:29132923

Franz MO, Chahl JS, Krapp HG (2004) Insect-inspired estimation of egomotion. Neural Computation 16:2245-2260

Graham P, Philippides A, Baddeley B (2010) Animal cognition: Multi-modal interactions in ant learning. Current Biology 20:R639-R640

Hempel de Ibarra N, Philippides A, Riabinina O, Collett TS (2009) Preferred viewing directions of bumblebees (Bombus terrestris L.) when learning and approaching their nest site. Journal of Experimental Biology 212:3193-3204

Jander R (1997) Macroevolution of a fixed action pattern for learning: The exploration flights of bees and wasps. In: Greenberg G, Tobach E (eds) Comparative Psychology of Invertebrates., Garland Publishing, New York, pp 7999

Kamikouchi A, Inagaki HK, Effertz T, Hendrich O, Fiala A, Göpfert MC, Ito K (2009) The neural basis of drosophila gravity-sensing and hearing. Nature 458:165-171

Koenderink JJ, van Doorn AJ (1987) Facts on optic flow. Biological Cybernetics 56:247-254 
Lehrer M (1993) Why do bees turn back and look? Journal of Comparative Physiology A 172:549-563

Lehrer M, Collett TS (1994) Approaching and departing bees learn different cues to the distance of a landmark. Journal of Comparative Physiology A 175:171-177

Linander N, Dacke M, Baird E, Hempel de Ibarra N (2018) The role of spatial texture in visual control of bumblebee learning flights. Journal of Comparative Physiology A 204:737-745

Lobecke A, Kern R, Egelhaaf M (2018) Taking a goal-centred dynamic snapshot as a possibility for local homing in initially naïve bumblebees. Journal of Experimental Biology 221:jeb168674

Murray T, Zeil J (2017) Quantifying navigational information: The catchment volumes of panoramic snapshots in outdoor scenes. PLOS ONE 12:1-24

Narendra A, Gourmaud S, Zeil J (2013) Mapping the navigational knowledge of individually foraging ants Myrmecia croslandi. Proceedings of the Royal Society B: Biological Sciences 280:20130683

Opfinger E (1931) Über die Orientierung der Biene an der Futterquelle. Zeitschrift für Vergleichende Physiologie 15:431-487

Philippides A, Hempel de Ibarra N, Riabinina O, Collett T (2013) Bumblebee calligraphy: the design and control of flight motifs in the learning and return flights of Bombus terrestris. Journal of Experimental Biology 216:10931104

Riabinina O, Hempel de Ibarra N, Philippides A, Collett TS (2014) Head movements and the optic flow generated during the learning flights of bumblebees. Journal of Experimental Biology 217:2633-2642

Robert T, Frasnelli E, Hempel de Ibarra N, Collett TS (2018) Variations on a theme: bumblebee learning flights from the nest and from flowers. Journal of Experimental Biology 221:jeb.172601

Samet N, Zeil J, Mair E, Boeddeker N, Stürzl W (2014) Ground-nesting insects could use visual tracking for monitoring nest position during learning flights. In: From Animals to Animats 13, Springer, pp 108-120

Srinivasan MV (1993) How insects infer range from visual motion. In: Miles FA, Wallman J (eds) Visual motion and its role in the stabilization of gaze, Elsevier, Amsterdam London, pp 139-156

Srinivasan MV, Zhang S, Altwein M, Tautz J (2000) Honeybee navigation: Nature and calibration of the "odometer". Science 287:851-853

Strübbe S, Stürzl W, Egelhaaf M (2015) Insect-inspired selfmotion estimation with dense flow fields - an adaptive matched filter approach. PLOS ONE 10:1-35

Stürzl W, Zeil J (2007) Depth, contrast and view-based homing in outdoor scenes. Biological Cybernetics 96:519-531

Stürzl W, Boeddeker N, Dittmar L, Egelhaaf M (2010) Mimicking honeybee eyes with a 280-degree field of view catadioptric imaging system. Bioinspiration \& Biomimetics 5:036002

Stürzl W, Grixa I, Mair E, Narendra A, Zeil J (2015) Threedimensional models of natural environments and the mapping of navigational information. Journal of Comparative Physiology A 201:563-584

Stürzl W, Zeil J, Boeddeker N, Hemmi JM (2016) How wasps acquire and use views for homing. Current Biology $26: 470-482$

Tinbergen N (1932) Über die Orientierung des Bienenwolfes (Philanthus triangulum Fabr.). Zeitschrift für Vergleichende Physiologie 16:305-334
Tinbergen N, Kruyt W (1938) Über die Orientierung des Bienenwolfes (Philanthus triangulum Fabr.). III. Die Bevorzugung bestimmter Wegmarken. Zeitschrift für Vergleichende Physiologie 25:292-334

Wagner W (1907) Psycho-biologische Untersuchungen an Hummeln mit Bezugnahme auf die Frage der Geselligkeit im Tierreiche. Schweizerbart'sche Verlagsbuchhandlung, Stuttgart

Zeil J (1993a) Orientation flights of solitary wasps (Cerceris; Sphecidae; Hymenoptera): I. Description of flight. Journal of Comparative Physiology A 172:189-205

Zeil J (1993b) Orientation flights of solitary wasps (Cerceris; Sphecidae; Hymenoptera): II. Similarities between orientation and return flights and the use of motion parallax. Journal of Comparative Physiology A 172:207-222

Zeil J (1997) The control of optic flow during learning flights. Journal of Comparative Physiology A 180:25-37

Zeil J (2012) Visual homing: An insect perspective. Current Opinion in Neurobiology 22:285-293

Zeil J, Kelber A, Voss R (1996) Structure and function of learning flights in ground-nesting bees and wasps. Journal of Experimental Biology 199:245-252

Zeil J, Hofmann MI, Chahl JS (2003) Catchment areas of panoramic snapshots in outdoor scenes. Journal of the Optical Society of America A 20:450-469

Zeil J, Boeddeker N, Hemmi JM, Stürzl W (2007) Going wild: Toward an ecology of visual information processing. In: North G, Greenspan R (eds) Invertebrate Neurobiology, Cold Spring Harbor Press, pp 381-403

Zeil J, Boeddeker N, Stürzl W (2009) Visual homing in insects and robots. In: Floreano D, Zufferey JC, Srinivasan MV, Ellington C (eds) Flying Insects and Robots, Springer, pp $87-100$ 